\title{
Nanothera(g)nosis and Chemistry: A Fruitful Binomial
}

\section{Miguel Valcarcel ${ }^{\star}$}

University of Córdoba, 14071, Córdoba, Spain

\begin{abstract}
The main purpose of this perspective article is to open the eyes of chemists to the great potential of Nanothera $(\mathrm{g})$ nosis, an emerging branch in Medicine, and the crucial roles that they can play in this promising topic in an interdisciplinary atmosphere. The present and future situation of Nanothera(g)nosis in the Nanomedicine field are critically presented and discussed in this minireview by distilling the most relevant insights of this relatively recent but hot topic. After a contextualization of it, the updated generalities of Nanothera(g)nosis are deal with. The most frequent thera(g)noastic agents participating in this process as well as the main types of Nanothera $(\mathrm{g})$ nosis processes according to several classification criteria are reported. A brief description of the potential impact of Nanothera $(\mathrm{g})$ nosis in several relevant diseases are also presented. The crucial roles of Chemistry in the two bottle-necks of Nanothera $(\mathrm{g})$ nosis namely the synthesis of isolated carrier nanothera(g)nositc agents and conjugated nanothera(g)nostic nanoparticles as well as the (bio)chemical analysis (i.e. quality assurance, monitoring of synthetic procedures and the characterization of final pharmaceutical drug) are outlined. Finally, some author's reflections about the future of this emerging nanotechnology included.
\end{abstract}

NANOTECHNOLOGY

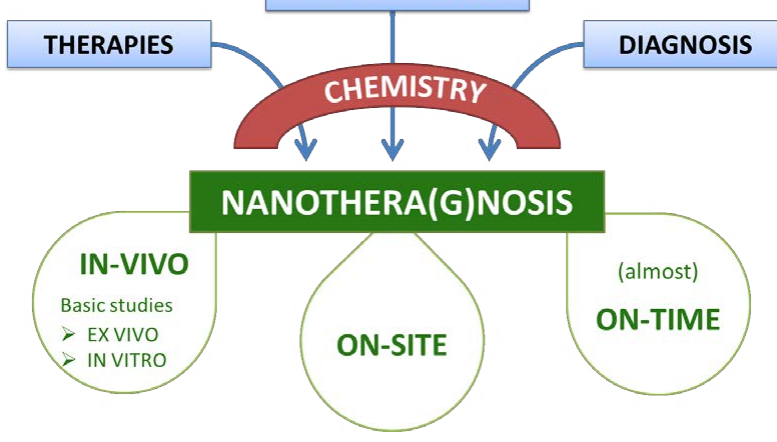

Keywords: Nanotheragnosis nanomedicine; Interdisciplinarity; Chemistry; Therapy; Diagnosis

\section{Introduction}

The impact of Nanotechnology in Medicine in a short period of time is undeniable. Nanomedicine [1] can be considered as a revolution in Health Sciences and Practices, according to very relevant organizations as. OMS, NIH-USA, which is reflected in the almost linear growing of research papers per year in scientific databases as well as the number of enterprises created to produce nanotools for medical purposes. No doubt, is the nanotechnological area of higher social repercussion and economic impact. The main objective of Nanomedicine is the development of a variety of nanotools to prevent, diagnose, and cure relevant diseases in its early stages (e.g. diabetes, cancer, Alzheimer, tuberculosis, infections, etc.) that show net advantages relative to the traditional medical tools owing to the exceptional physic-chemical characteristics of nanomatter. There are several possibilities to describe basic advances and realities of Nanomedicine. One of them is based on medical specialities (e.g. nano-oncology, nano-cardiology, nanonephrology, nano-dermatology, nano-surgery, nano-traumatology, etc.); this classification is not suitable since it establishes artificial "water thigh compartments". The classification proposed in Figure 1 is more transversal and realistic; it is based on the function of nanotools in Medicine:

- Nano-regenerative medicine based mainly on tissue regeneration using nanotecnological scaffolds [2], which show clear advantages as regards the conventional ones;
- Nano-diagnostics: analysis and/or bioimaging, in vivo, ex vivo and in vitro in order to detect and monitor the evolution of diseases [3];

- Nano-therapies mainly based on drug delivery by using nanoparticles [4]. There are other atypical nano-therapies such as nanotecnological-based artificial implantable kidneys [5];

- Nano-thera(g)nosis, the subject matter of this article, which is a combination of nano-therapies and nano-diagnosis, as is shown in the graphical abstracts;

- Nano-asepsis oriented to the efficient prevention and elimination of pathogens in hospitals [6].

In addition, there are other minor branches of Nanomedicine, but also of great practical interest. Such is the case of the use of nanocellulose to reinforce latex, named as nano-latex, that enables the production of nanogloves for clinical purposes with a reduced thinness and high

*Corresponding author: Miguel Valcarcel, University of Córdoba, 14071 Córdoba, Spain, Tel: 34670667870; E-mail: qa1vacam@uco.es

Received: March 13, 2018; Accepted: March 27, 2018; Published: March 30 2018

Citation: Valcarcel M (2018) Nanothera(g)nosis and Chemistry: A Fruitful Binomial. J Nanomed Nanotechnol 9: 490. doi: 10.4172/2157-7439.1000490

Copyright: (c) 2018 Valcarcel M. This is an open-access article distributed under the terms of the Creative Commons Attribution License, which permits unrestricted use, distribution, and reproduction in any medium, provided the original author and source are credited. 


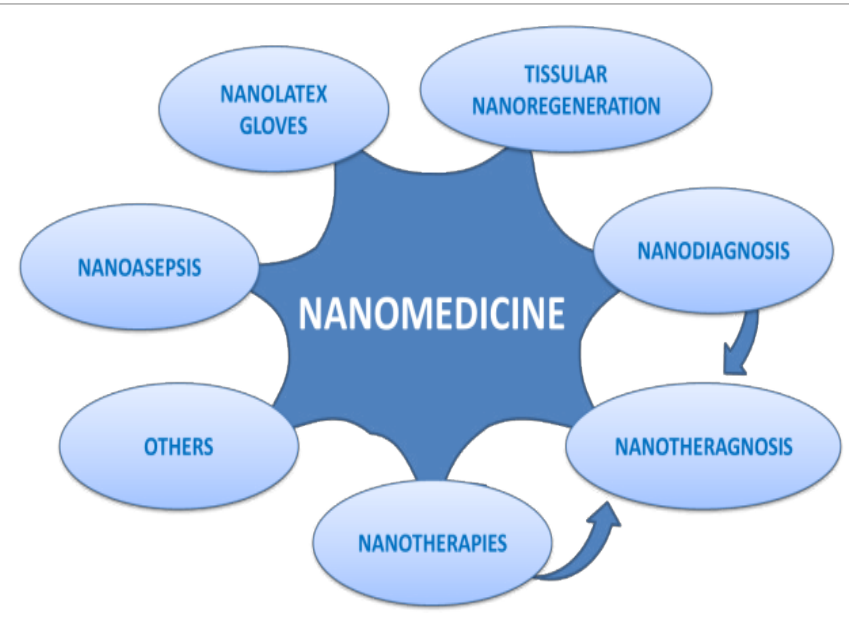

Figure 1: Transversal classification of Nanomedicine according to the main nanoactivities in each medical specialty (e.g. cardiology, oncology, nephrology, rheumatology, traumatology, microbiology, etc.).

breaking strength [7]. The two above-mentioned Nanomedicine classifications are not in contradiction. For example, nanonephology encompasses renal nanodiagnostics, renal nanotherapies, renal nanothera(g)nostics, growing renal cells in nanotechnological-based scaffolds, among others. Nanothera(g)nosis is, thus, one of the most promising branches of Nanomedicine. In a few years, the growing number of developments and publications on this topic is spectacular. It is interesting to note that thera(g)nosis was proposed in the $20^{\text {th }}$ century. But the revolution of the use of nanoparticles as carriers in thera(g)nosis has deeply changed the combination of therapies and diagnostics, as is recognized by the majority of clinical researchers.

According to the scientific international databases (i.e. WOS, Scopus), "chemistry" is the area of knowledge most associated with "nanotechnology". In "nanomedicine" realm, "chemistry" is the second one after "medicine". The transversal character of chemistry is ideal and useful for interdisciplinary studies and applications. According to Whitesides [8], being in the frontiers of knowledge together with a variety of areas is the most promising avenue of Chemistry in the $21^{\text {th }}$ Century.

In this paper, a new, objective and general vision of Nanothera(g) nosis is presented taking into account the experimental papers, revisions,, reports, recent books [9-11] and, even, a specific journal [12] published until now. All of them constitute a good proof of the consolidation of this tendency of Nanomedicine and its promising capabilities and realities. The general features, the agents involved in the process, the main types of agents involved as well as some examples of the use of Natotera(g)nosis to efficiently fight specific diseases in the early stages are described. The present roles of Synthetic and Analytical Chemistry are dealt with in a systematic way. Finally, the opinion of the authors about the future of Nanothera(g)nosis is also included.

\section{Generalities of Nanothera(g)osis}

There are many approaches describing nanothera(g)nosis in the literature. Almost all of them emphasize particular aspects. The introduction to the topic in this paper is a summary of almost all the connotations that converges in it. This item includes a global definition, the objectives, the nomenclature and its interdisciplinary character.

\section{Definition}

The graphical abstract of this article represents a general schematic definition of Nanothera(g)nosis: a combination of external (i.e. energies) and/or internal (e.g. drugs) therapies and diagnostics for different purposes such as bioimaging, DNA beacons to direct external energies to the target site, monitoring the efficiency of therapies, among others. All of them with the support of nanotechnology. Nanothera(g)nostic processes can be globally described as the intake of the nanotheragnostic drug by injection in the bloodstream or as pills. The thera(g)nostic agent is transported to the specific site with the aid of targeting ligands and then the processes starts on site.. The three basic characteristics of them arising from how are implemented are:

- "On-site", that is, in the well-defined target place in the human body (e.g. in a lung tumour).

- Almost "on time", because there is an implementation order: first therapies and second diagnostics. In some cases both processes takes place simultaneously. It is also possible in the reverse order: diagnostics and therapies.

- "In vivo", that is in animal and human bodies. Previous basic studies have performed both "ex vivo" (experiments implemented in tissue/organ extracted from a living organism) and "in vitro" (artificial experiments in research laboratories simulating biological systems).

The main contribution of nanotechnology is the involvement of nanoparticles as carriers, which implies clear advantages as regards the classical Thera(g)nosis.

\section{Objectives}

The strategic importance of nanotera(g)nosis arises from its ambitious objectives, that can be classified into three groups according to complementary criteria, namely:

General objectives included in the definition, such as the combination on site on time, in vivo (ex vivo and in vitro) of therapies and diagnostics monitoring the efficiency of therapies involved, among others;

Technical objectives such as miniaturization (owing to nanosize), simplification (owing to integration of actions), efficiency (owing to the target drug delivery and release), among others;

Clinical objectives, which are the most relevant:

- Minimization of the use of drugs (i.e. chemotherapy agents) and, in this way, the drastic reduction of the hard side effects;

- Smart and efficient selection of the appropriate drug for the specific disease;

- Personalized medicine: control of new drugs, design of specific clinical treatments, etc.; and

- Reduction of waiting time in hospitals.

These clinical objectives are related among them.

\section{Interdisciplinary character}

Nanothera(g)nosis is a very representative topic of those were is essential, mandatory, of breaking the frontiers of isolated areas. This confluence can be described in two main ways. The simplest one is the combination of therapies, diagnostics and nanotechnology [13]. A more complete approach to the interdisciplinary character of Nanothera $(\mathrm{g})$ 
nosis is the combination of Medicine, Chemistry, Biology, Pharmacy, Nanotechnoloy, and Bioimaging [14].

\section{Nomenclature}

The original term, "nanotheragnosis" has increasingly been superseded by "nanotheranosis", probably because the latter is easier to pronounce. However, dropping the "g" was a strategic error because "diagnosis" almost disappeared from the title. The author proposes recovering the Greek root "gnosis" to signify the information or knowledge provided by the act of diagnosing.

One other linguistic issue is the correct use of "thera(g)nostic" (whitout s) as an adjective [e.g., in "thera(g)nostic agents] and of "thera(g)nostics (whict s), to refer to the art or discipline to combine therapies and diagnosis as "Mathematics!.

The author propose one way to circumvent these problems which is the standardization of the previous terms in the form of an global acronym such as NTD (Nano-Therapy-Diagnostics).

\section{Nanothera $(g)$ tic agents}

They can be defined as chemical, biochemical, immunological and gene species that implement complementary functions in the naothera $(\mathrm{g})$ nostic process. It is possible to distinguish two types.

The essential nanothera $(g)$ nostic agents are:

- Nanocarriers that transport other agents;

- Diagnostic agents;

- Therapeutical agents; and

- Targeting ligands that direct the nanocarrier to the specific disease site; in some cases, they are not necessary.

The complementary agents are those that facilitate the functions of the main agents. Such is the case of DNA beacons with fluorophores to enable the external therapy, antifouling and anti-aggregation ligands (i.e. polyethileneglycol, human serum albumin) at the surface of the nanocarrier.

\section{Types of Nanotera $(g)$ nosis: Clasifications}

The abrupt growing of Nanotera(g)nosis, reflected in the impressive number of publications in the last years, has led to the description of a great number and variety of these promising processes. To face some systematization of the topic, it is necessary to take into account several classification criteria, the most relevant of which are depicted in Figure 2 . These criteria are not contradictory but complementary in such a way that they can be used to fully define a nanothera(g)nostic procedure.

\section{Nanoparticles (carriers) involved}

The use of nanoparticles as carriers is the key aspect of the Nanothera(g)nosis processes and the main reason of the great impact of these in fighting diseases. There are several sub-classifications according to several sub-criteria, namely:

- According to nature of the nanocarrier, it can be inorganic (i.e. gold nanoparticles, nanomagnetite, silver nanoparticles, etc.), organic (i.e. dendrimers, liposomes, carbon nanoparticles, nanoestructured polymers, etc.) and biological (e.g. biopolymers, etc.).

- According to the compactness of the nanocarrier, it can be

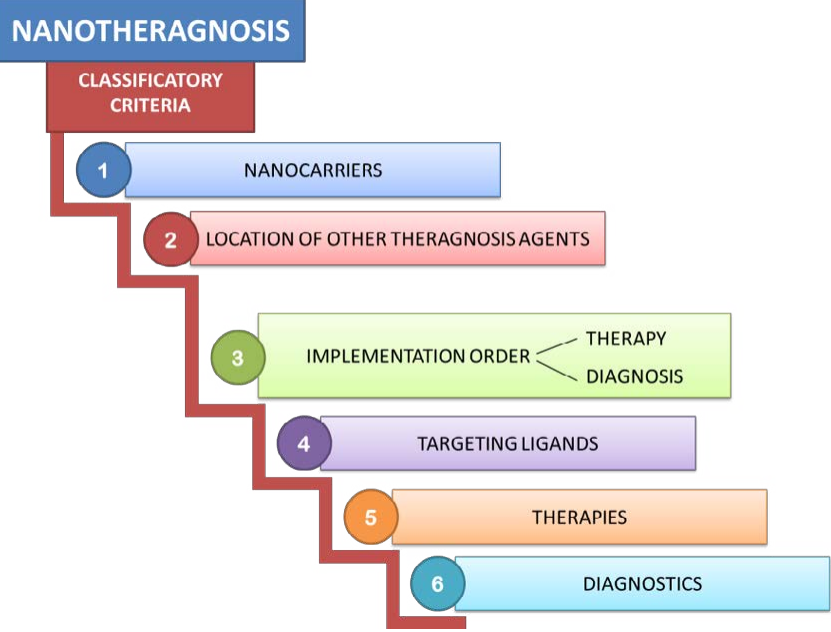

Figure 2: Description of six complementary classification criteria to fully describe nanoteragnostic processes. For details, see text.

fully compact (e.g.gold nanoparticles), porous (e.g. organic and biological polymers) and hollow (e.g. liposomes).

- According to the external shape of the nanocarrier, it can be a rod, a sphere, a triangle, a star, among other less common forms.

- According to the number of different nanocarriers involved, the most usual situation is when a single nanocarrier transport all the thera(g)nostic agents. In some cases, two different nanocarriers are involved (i.e. one transporting the therapeutic agent and the other the diagnosis agent).

\section{Location of other thera $(\mathrm{g})$ nosis agents at the nanocarriers}

There are three possibilities regarding the incorporation of thera $(\mathrm{g})$ nostic agents at the nanocarrier, namely: A) At the surface (e.g. red gold nanoparicles decorated with antibodies, targeting agents, DNA-based nanobacons [15-21], antifouling/anti- aggregating agents, among others); B) Inside of hollow or porous nanoparticle (e.g. dendrimer transporting both therapeutic and diagnostic agents); and C) Inside and outside of the nanocarrier (i.e. liposome containing the therapeutic and diagnostic agents inside and the antifouling and targeting ligands at its surface of it).

\section{Implementation order of therapies and diagnoses}

This is an atypical classification little recognized in the abundant literature describing nanohera $(\mathrm{g})$ nosis processes. The most frequent situation is when the therapy is the first step and the diagnosis of its effectiveness, the second. But the reverse situation can also be found such is the case of a nanothera $(\mathrm{g})$ nostic agent for diabetes in which the external membrane of the nanocarrier is broken and delivers internal insulin if the concentration of glucose increases in blood [22].

\section{Targeting thera $(\mathrm{g})$ nostic agents}

They are ligands at the surface of the nanocarrier that actively direct it in the bloodstream to the disease zone of the human body (e.g. organ affected by a tumor) where they are anchored before starting the nanothera $(\mathrm{g})$ nostic process. The most frequent vectoring agents are antibodies and folic acid that are attached to antigens and folates of the target site, respectively. There are other directional ligands such as aptamers (oligonucleotids) and peptides. Their active function is essential in the majority of nanothera(g)nostic processes. 
On the other hand, passive targeting is based on the fact that vessels surrounding tumors are leaky since the endothelial lining are incomplete allowing nanotheragnosistic agents to reach the tumor cells through the enhanced permeability and retention (EPR) effect. Ordinarily, a combination of active and passive targeting takes place.

\section{Nanothera(g)nostic therapies}

There are several sub-criteria to classify the great variety of therapies involved in the theragnostic processes. The most relevant are as follows.

According to the origin of therapies, they can be internal and externalas regards the body. The internal therapies are those arising from the transportation of therapeutically drugs at/in the nanocarriers. External therapies are those based external energetic sources; being lasers and ultrasounds the most frequent ones. In this case the nanothera(g)nostic process should involve beacons to mark (imaging) the target disease zone (e.g. a tumor) in order to direct the external energy source to it.

According to the basic therapy types, there are chemical, genetic, photonic therapies, among others involved in the process of nanothera $(\mathrm{g})$ nosis.

According to the number of therapies involved in a single nanothera(g)nostic process, the most frequent situation is the use of a single therapy. On the other hand, several therapeutic strategies in the same process can be combined looking for synergistic effects. Such is the case of the combination of internal chemotherapies and external photonic energy transformed into thermal energy by nanocarriers in order to accelerate the chemotherapeutic effect [20].

Therapies could take place in the nanothera $(\mathrm{g})$ nostic process before or after the diagnosis (see part 4.3 of this paper).

\section{Nanothera(g)nostic diagnostics}

Diagnostics is a key process in nanothera(g)nosis because it is the source of information/knowledge for different purposes:

- To delimit the targeting zone if external therapies are involved;

- To monitor the effectiveness of the applied therapies;

- To indicate that therapies are needed when the diagnosis takes place is the first sub-process (see reversed nanotheragnosis, item 4.3 of this paper);

- To distinguish between "drug delivery" and "drug release" by bioimaging [14]

- Others, less general.

There are many types of diagnosis involved in the nanothera $(\mathrm{g})$ nostic processes, the most relevant of which are classified using complementary criteria, namely:

According to the instrumental technique that provides primary data, it can be nuclear magnetic resonance (NMR), spectroscopic fluorescence, ultrasonic energy, computed tomography (CT), positron emission tomography (PET), magnetic resonance imaging (MRI), among others. All of them are used for bioimaging of the target zone.

According to the number of diagnosis involved in a single nanothera $(\mathrm{g})$ nostic process, the most usual situation is when a single source of information is involved. In some instances, two or more diagnosis could take place; such is the case of the use of carbon quatum dots CQDs) derivatized with transition metal ions such as europium, manganese, gadolinium, as nanotheragnosis nanoparticles, that allows implementation of both fluorescent imaging from CQDs and MRI from the transition metals of Hela cells [16].

According to the time when the diagnosis process takes place, it can be distinguished between after (the most frequent situation) and before the therapy process (see item 4.3 of this paper)

According to the role of the nanoparticle in the diagnostics subprocess, it can be indicator (i.e. Semiconductor Quantum Dots derivatized with other theragnostic agents) or not (i.e. macroporous polymeric nanoparticles)

\section{Nanotera(g)nosis for Specific Diseases}

Several relevant examples of in vitro, ex-vivo and in vivo applications of nanotheragnostic processes are described here with the aim to demonstrate their potential to successfully treat and cure relevant diseases in their early stages.

Cancer, in general, is the more treated disease by nanothera $(\mathrm{g})$ nosis processes. An indicator of the importance of this focused topic is the fact a book on cancer nanothera(g)nosis has been published [17] as well as a recent critical review [18]. The fact that there are specific sites (tumors) in human bodies and the existence of specific antigens in the surface of tumor, are the key for the successful oncologic treatments using nanothera $(\mathrm{g})$ nogtics. Sengupta et al. recently described (Figure 3) a polymeric porous nanoparticle that transports internally both the therapeutical and diagnostic agents and the other agents such as directional folic acid, and antifouling polyethilene glycol (PEG) at the surface; when the tera(g)nostic nanoparticle reaches the folate sites of the tumor, the chemotherapy stars and the fluorescent indicator react with the caspase enzyme produced in the cellular cancer death and, in this way, monitors the efficiency of the therapeutic agent for a specific tumor. P. Pedrosa et al. [19] developed a very interesting nanothera(g) nostic process for renal cancer which is schematically depicted in Figure 4; at the surface of a red gold nanoparticle (15 nm of size), a directional antibody, DNA nanobeacons conjugated with a fluophore and a chemotherapeutic drug are attached; when the nanothera $(\mathrm{g})$ nostic nanoparticles arrive at the tumor in the kidney, the drug starts the chemotherapy and flurescence appeared owing to the fact that the nanobeacons are separated from the gold nanoparticle that act as a fluorencence quencher, this signal is being used to focuses an external laser beam to increase the tumor temperature $\mathrm{Up}$ yo $45^{\circ} \mathrm{C}$ ); in this particular case three therapies are conjugated: two internal (drug, gene) and one external (optical/thermal). There are many other approaches to nanothera(g)nostics for cancer diseases, such as lung cancer $[20,21]$.

Care of patients of diabetes (type 1 and 2) diseases can be clearly improved by the use of nanotechnology [22]. Nanoparticles can act as imaging agents, implanted electrochemical sensors, among other functions Probably, the most interesting nanodiabetes approach is the use of glucose-responsible nanoparticles in the nanothera(g)nosis context, that mimics the pancreas function: insulin delivery when the concentration of glucose raises in the bloodstream (Figure 5). The nanoparticle involved consists of an external biopolymer cover that reacts (swelling or breaks) when the concentration of glucose increases and insulin located at it is delivered to the bloodstream. This is an atypical nanothera(g)nostic process because: A) It is an example of the less frequent reversed order: diagnostic and therapy; B) There is not a target site in the organism because the process takes place in all the bloodstream; and C) Directional ligands are not involved (Figures 6 and 7). 

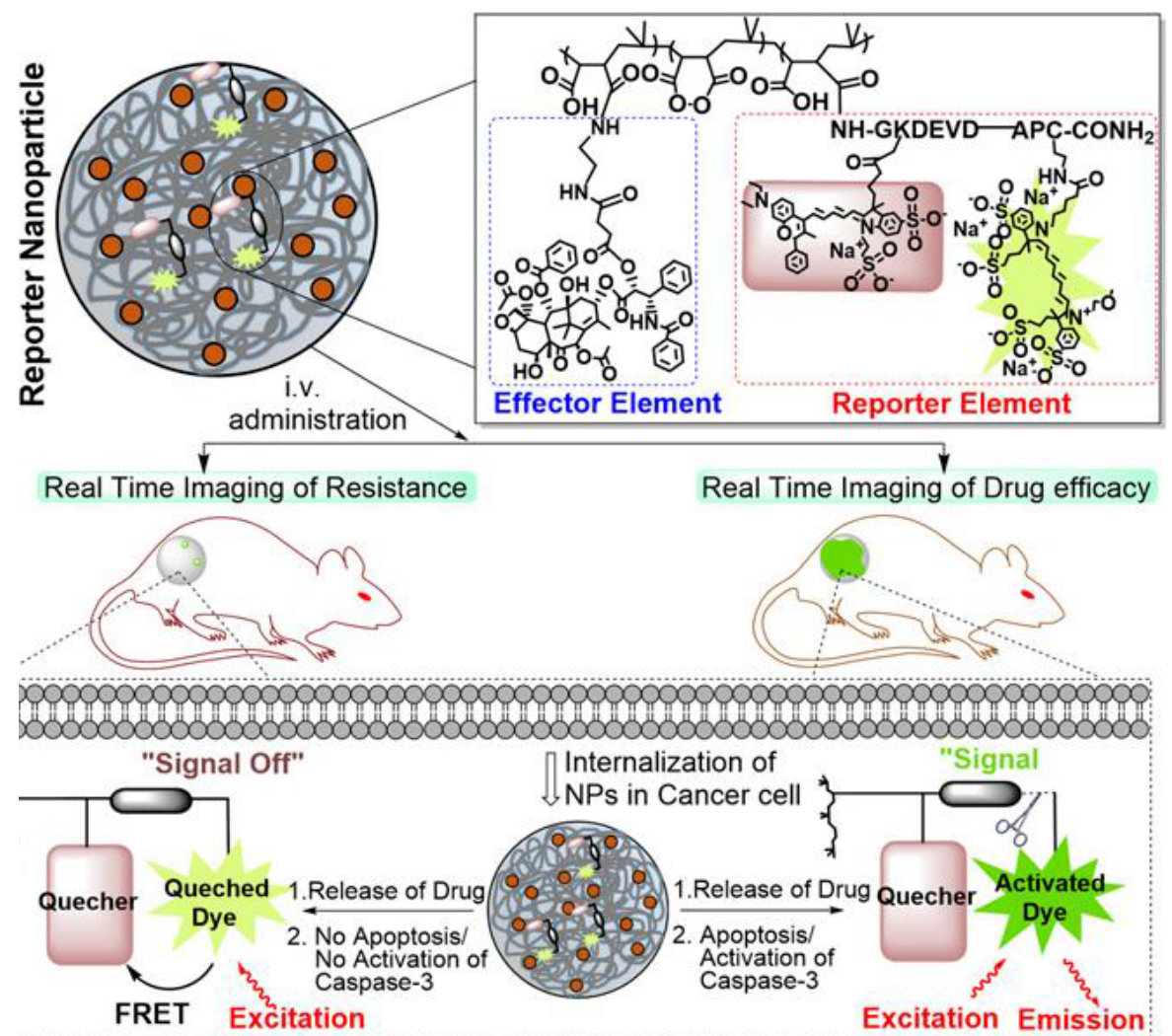

Figure 3: On site diagnosis after therapy in a nanotheragnostic process using a polymeric nanoparticle that transports the chemotherapeutic and the fluorescent diagnostic agents to the cancer tumor to pointed out the presence of the caspase enzyme, which indicates cellular death. From reference 19, with permission of National Academy of Sciences (USA).

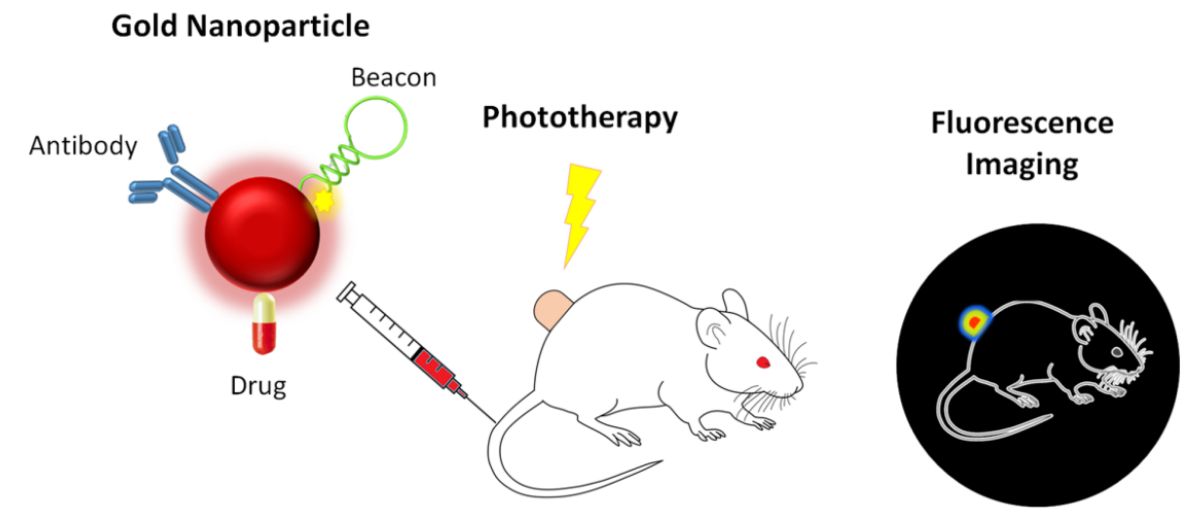

Figure 4: Schematic diagram of the use of a red $(15 \mathrm{~nm}$ of diameter) gold nanoparticle as carrier transporting the chemotherapeutical, directional, diagnostic and antifouling agents. A suspension of it is injected to the animal and the fluorescence directs and additional laser therapy to the tumor. From reference 20 , with permission of MDPI AG (Basel).

Alzheimer disease has been the target of several nanothera(g) nostic strategies mainly oriented to combat and control the formation of the typical brain plaques caused by the fibrilar amyloid- $\beta$ peptide $(\mathrm{A} \beta)[22,23]$. One strategy $[24]$ is the use of specific anti-monoclonal antibodies to delay the aggregation of fibrilar $A \beta$, but they should be frequently supplied. The immobilization of $A \beta$ at the surface of a near infrared (NIR) fluorescent iron oxide nanoparticle circumvent this problem and opens up a great potential to treat neurodegenerative diseases; in this case, no targeting agents are necessary and the dual diagnostics is performed by fluorescent and magnetic resonance imaging both in vitro and in vivo. Busquets et al. have reviewed the use of magnetic nanoparticles to detect and treat Alzheimer disease. The fact that some carbohydrates as gangliosides play a relevant role in the early steps of aggregation of $A \beta$ stimulated Kouyoumdjian et al. [25] to synthesize conjugated iron oxide gluconanoparticles to detect $\mathrm{A} \beta$ in vitro and ex-vivo by $\mathrm{MRI}$; in addition, the nanothera(g)nostic nanoparticle greatly reduced $A \beta$ induced cytotoxicity to cells.

Bacterial infections of pathogens such as bacteria in specific sites of the human body have been also successfully treated and diagnosed 


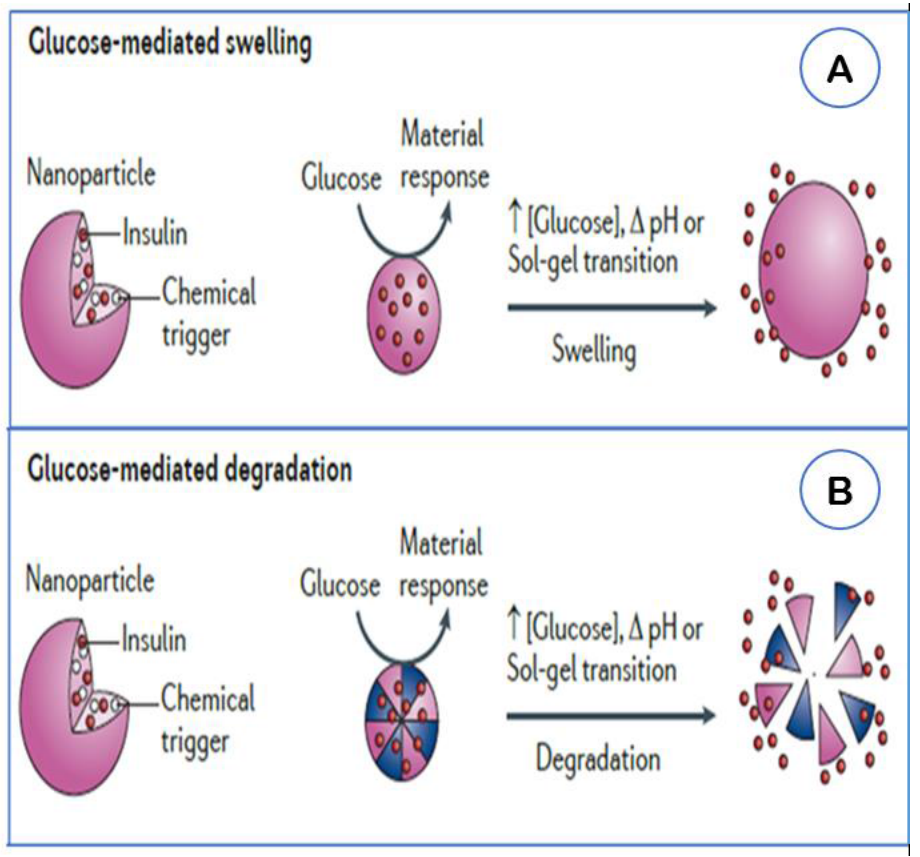

Figure 5: Example of the reversed implementation order (diagnosis and therapy). The theragnostic nanoparticle is composed by a biopolymeric membrane that swelling $(A)$ or break $(B)$ in the bloodstream when the glucose concentration increases. The internal located insulin is delivered as result.

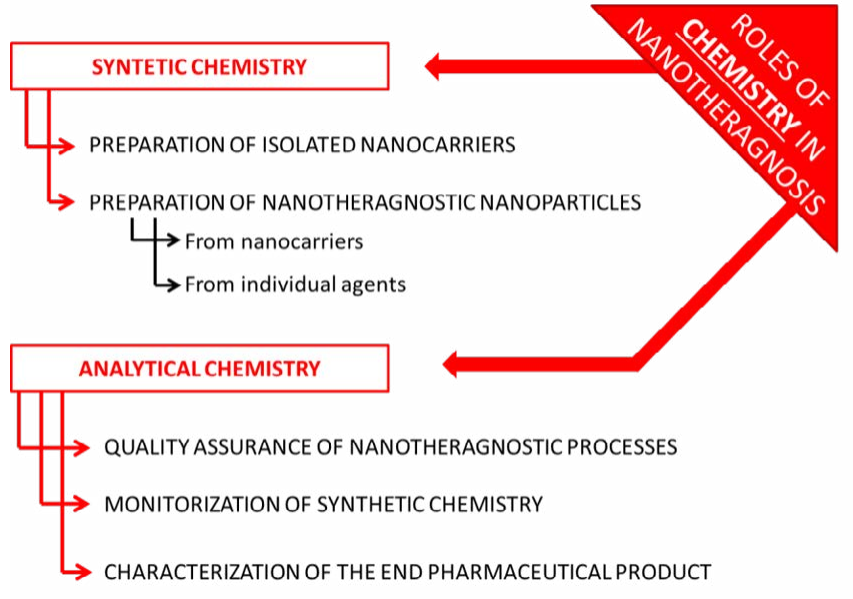

Figure 6: Schematic diagram of the crucial roles that can play Chemistry in nanotheragnosis.

by nanothera(g)nostic processes. For example, Kim et al. [26] use of clusters of single-walled carbon nanotubes which have a high affinity for bacteria (i.e. E. Coli) for laser photothermal therapies and diagnosis of the effects of therapies using transmission electron microscopy (TEM) and phototheray (PT). It is interesting to note that in this case, there are no targeting agents involved [27].

There are other diseases that have been the target of nanothera $(\mathrm{g})$ nosis processes. Among them, tuberculosis [28], ischemic and hemorrhagic strokes [29] and liver disorders [30] are the more relevant.

\section{The Strategic Roles of Chemistry in Nanotheragnosis}

The global role of Chemistry in nanothera(g)nostic processes is depicted in the graphical abstract of this paper. It is essential in

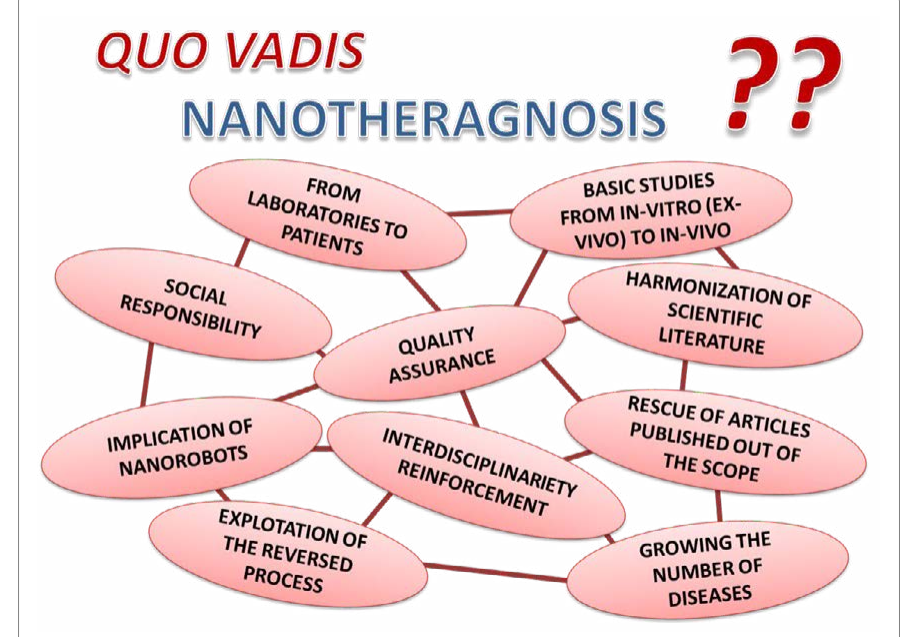

Figure 7: Foreseeable trends in Nanothera(g)nosis.

therapies and diagnostics supported by nanotechnology. Chemistry is a great enabler according to Matlin et al. [30]. This item schematizes the opportunities and challenges of chemists in a very open topic now, when the innovation-interdisciplinary train is still passing through the railway station of Chemistry,

Anyway, the activity of Chemistry in Nanothera(g)nosis in order to be efficient must be carried out in a interdisciplinary atmosphere (see item 2.3 of this paper), particularly with biochemistry, pharmacy and bioimaging areas.

Chemistry can play a decisive role to support the evolution of nanotheragnostic processes from in vitro to in vivo, which is one of the main week points of Nanothera(g)nosis. 
The specific roles of Chemistry are commented on herein. A distinction is made between Synthetic and Analytical Chemistry, two of the vortexes of the so called "Basic Triangle of Chemistry" in addition to the other vortex (Theory). The fourth vortex of the so named "Chemistry tetrahedron" is Applications [31,32] that, as background, is also involved in this context.

\section{Synthetic chemistry}

The fact that several books on tailored nanomaterials for therapies, diagnostics and thera $(\mathrm{g})$ nostics have been recently published $[10,11]$ is a proof of the importance of the topic. But there are many unexplored synthetic approaches that can be deal with in the next years. In many instances, the biochemical approach is mandatory.

It is possible to distinguish between the preparation of isolated nanocarriers as a previous step before the attachment of other thera $(\mathrm{g})$ nostic agents and the synthesis from the isolated raw materials that are components of the nanother $(\mathrm{g})$ nostic nanoparticle.

Synthesis of isolated nanocarriers: Nanoparticles $(1-100 \mathrm{~nm})$ can be fabricated by two main ways according the raw products. In the "top-down" approach, a bulk material at the macroscopic/microscopic level is reduced to the nanoscale by using mechanical, physical or chemical techniques. In the "bottom-up" approach atoms or molecules of subnanometric size are assembled through chemical reactions and/ or physical processes to generate a nanomaterial. Another relevant aspect of the synthesis of nanoparticles is the nature of the nanoparticle which can be inorganic, organic and biological.

Inorganic nanocarriers are of pure metals or their oxides; the most relevant involved as nanocarriers in nanothera(g)nostic processes are commented on.

Among the different methods described for the synthesis of gold nanoparticles, they are most often "bottom up" prepared by reduction of soluble gold halides (e.g. $\mathrm{HAuCl}_{4}$ ) with the use of chemical reducing agents in solution such as sodium citrate or sodium borohydride, among others, which usually requires the use of further stabilizers or ligands. Recently, a simple, quick and cost-effective approach to prepare bare gold nanoparticles without any ligand on their surface has been described, which is based on the use of stainless steel as solid reducing agent, yielding stable AuNPs at atmospheric pressure and room temperature within a few minutes [33]. Both approaches and others allow the control of the size, and hence the color, of gold nanoparticles [34].

Synthesis of magnetic iron oxide nanoparticles are also very relevant in Nanothera(g)nosis because they facilitate the diagnostic step (e.g. using MRI). Different core-shell inorganic nanoparticles with tunable size, such as magnetite as core and gold as a shell can be onepot prepared by almost simultaneous gold formation and attachment onto the magnetite core surface via a S-Au covalent bonding. This nanocomposite is demonstrated to have a great potential for (MRI)guided, focused photothermal tumor therapy under near-IR laser radiation [35].

Nanostructured porous silica particles (crystalline $\mathrm{Si}$ that is traversed by nanometer-width pores, providing the material a high surface-to-volume ratio) are widely used to transport both therapeutical and bioimaging agents because their low toxicity and biodegradability in the organism; they are fabricated by a top-down approach from a bulk material using a wide range of methods, including chemical stain, chemical vapor, laser-induced etching and metal-assisted etching processes, spark processing, electrochemical anodization and reactive ion (plasma) etching [4]

Organic nanocarriers are very attractive in nanothera(g)nostics thanks to their high renal clearance and reduced toxicity as regards the inorganic ones. There are many types of these nanocarriers involved in nanothera(g)nosis, the most relevant are commented on.

Dendrimers are prepared in isolation using different synthetic routes [36] but they are scarcely prepared in this way for their use as nanocarriers. The most frequent situation is the synthesis of conjugated metallic nanoparticles and dendrimers (see item 8.1.2).

Biopolymers are an emerging alternative of nanocarriers for thera $(\mathrm{g})$ nosis owing to their sensibility to external molecules. Such is the case of molecularly imprinted nanobiopolymers synthesized using glucose and phenylboronic acid which, through reversible hydrogen bonding interactions, form supramolecular assemblies that breaks or swelling in the presence of glucose. This is one of the mechanisms of the nanothera(g)nosis of glucose (Figure 5) [22].

Liposomes are prominent nanocarriers for delivering of hydrophobic and hydrophilic drugs, diagnostic agents, peptides, antibodies, etc after their encapsulation in the internal volume of a concentric bilayer of natural and synthesized phospholipids. There are a great variety of preparation techniques of liposomes (e.g. physical dispersion using or not sonication, membrane extrusion, $\mathrm{pH}$-induced, etc.), but the majority of them in the presence of the molecules to be encapsulated (see item 6.1.2). Isolated preparation of liposomes, are scarcely used in nanothera $(\mathrm{g})$ nosis processes.

Synthesis of conjugated thera(g)nostic nanoparticles: The formation of nanothera(g)nosis particles can be approached by using: A) Two step preparation from previously synthesized nanacarriers to which the other agents are internally or externally incorporated; or B) Several steps preparation from the individual components of the final nanothera(g)nosis particles. There are other possibilities but lees common.

Preparation from isolated nanocarriers is the most frequent situation when they are of inorganic nature. It is a multiple synthetic route. Such is the case of decorated gold nanoparticles (Figure 4) [20] for cancer nanothera(g)nosis. The $15 \mathrm{~nm}$ red gold nanoparticles are prepared using the usual reduction method. It is well known the easy surface functionalization of gold nanoparticles with chemotherapeutic agents such as 5-fluorouracil [37], vectoring ligands as antibodies [38], antifouling/anti-aggregation agents as PEG and nanobacons based on herpin ssDNA structures derivatized with fluophores [20]. Also, it is usual that nanostructured porous silica nanoparticles are first prepared using the synthetic routed defined above and then, the other thera $(\mathrm{g})$ nosis agents are incorporated both inside the nanopores (i.e. therapeutic and imaging drugs) and the active targeting and antifouling agents in their surface [4].

From individual thera(g)nostic agents In this case, synthesis of nanacarriers involves also the incorporation of the other thera $(\mathrm{g})$ nostic agents in the nanoparticle using a single step procedure, that can be named as encapsulation procedure. This is the typical situation when organic nanocarriers are involved. Such is the case of nanothera(g)nosis-based on liposomes as nanocarriers, being possible to encapsulate hydrophobic quantum dots within the phospholipid bilayers via micelle-to-vesicle transition approach to enhance the optical characteristics of isolated quantum dots [39]. 


\section{Analytical chemistry}

Among others, several global tendencies of analytical chemistry (bioanalysis, direct analysis, simplification, interdisciplinarity) are involved in the roles of this discipline of chemistry in nanothera $(\mathrm{g})$ nosis. In addition, the dual relationship between Nanotechnology and Analytical Chemistry has been recently reviewed.

In this context it is necessary to distinguish between monitoring (generation of information) and control (embracing monitoring and making decisions).

The most relevant analytical activities in nanothera(g)nostic processes are commented on below classified in three interrelated main groups of analytical actions, namely:

Quality assurance of the R\&D\&T in Nanothera(g)nostic processes: To guaranty the reliability of punctual and bioimaging results it is mandatory to systematically apply quality assurance methodologies in all processes involved in nanothera(g)nostics [40]. There are few articles on Nanothera(g)nostics in which the results of bioimaging are repeated few times, and the average is presented [24]. This approach is the minimum level but clearly not enough.

A very interesting critical review on the state of the art and prospects of non-invasive imaging in Nanomedicine and Nanotheranosis involving a great variety of instrumental techniques has been recently published [41], but the calibration of the instrumentation is forgotten as is the use of reference materials to assure their correct behavior. Instruments and sensing systems always provide data; the challenge is to be sure that these experimental data correspond to the reliality.

The lack of the systematic application of quality assurance systems in bioanalysis (e.g. genomics, proteomics) should be circumventing. The involvement of analytical chemists in interdisciplinary teams is essential in this respect.

An additional challenge is transforming primary data (e.g. fluorescent intensity, images) to results (information) and to knowledge (reports). In this sequence, experienced analytical chemists plays an important role together with other scientists from other areas, and, in this way, the scientific decisions will be timely and well founded [42]. The interdisciplinary approach is one of the main opportunities and challenges of Analytical Chemistry [43].

Monitoring synthetic chemistry: The quality control of raw, intermediate and end products in the synthetic processes involved in the preparation of nanothera(g)nostic agents is frequently forgotten. The added value of analytical chemists in the preparation steps is undeniable.

Batch-to-to batch reproducibility, in the framework of "research reproducibility" described by Ionidides et al. [44], is an essential aspect that should be systematically taken into account. In this way, it would be possible to reproduce the experimental procedures described in the publications which is unfortunately rare, in general. It is necessary to pursuit transparency in research articles. If the authors like to be opaque, they should explore the possibility to patent their contribution but avoiding the corresponding obscure paper.

Characterization of the final pharmaceutical product: The nanothera(g)nostic pharmaceutical product to be injected or pill inserted into the human body is the final tangible result of the nanotera(g)nostic research, development and transference (R\&D\&T) processes. Due the fact that there are few in the market, the characterization of it has not been systematically considered until now. The analysis (characterization) of it is a challenge in Nanothera(g)nosis which should be under responsibility of analytical chemists. There are two principal characterization lines:

- Homogeneity of batches related to the so called "batch-tobatch reproducibility".

- Storage (shelf) and in-use (once opened and the first aliquot is removed) stability. Stability tests are oriented to provide evidences of that active and exicipient substances vary or not with the time under the influence of temperature, humidity and light among other environmental factors.

Characterization of end nanothera $(\mathrm{g})$ nostic products implies many routine analysis, that should be done under two umbrellas: quality assurance and an experienced analytical chemist team.

\section{Summary}

\section{Quo Vadis, Nanothera(g)nosis?}

To finish this overview, a rapid overview of the future of Thera $(\mathrm{g})$ nosis is advisable. This perspective is materialized in a Decalogue of foreseeable directions:

- Evolution of basic studies on Nanothera(g)nosis: from "in vitro" and "ex vivo" to "in vivo" (animals, humans). On the other hand, ethical problems can be derived when passing from non-human to human in vivo live (see item 10 Social Responsibility).

- Acceleration of the process of transference of knowledge (from the laboratories to patients) following the sequence R\&D\&T Al these steps should have a high degree of true innovation. In other words, clinical research should be oriented to prevent and cure efficiently diseases and be far from the blue-sky research [45], but taking into account Social responsibility [10].

- Reinforcement the interdisciplinary character. If Nanomedicine is an new branch of Medicine with a clear interdisciplinary character, the confluence of scientific and technical areas such as Medicine, Chemistry, Biochemistry, Engineering, Physics, Pharmacy, Imaging etc. in Nanothera(g)nosis, is more notorious.

- Clarification of nomenclature. Authors propose here to use "Nanotheragnostics" because it better describes the two processes involved. The inclusion of "g" is essential to outline that diagnoastics is involved. Also the NTD (Nano-TherapyDiagnostics) acronym is proposed to support an efficient scientific and technical communication.

- Amplification of the diseases to be successfully treated with nanothara $(\mathrm{g})$ nostic processes.

- Exploitation of the reverse situation in nanothera(g)nosis : first diagnosis, and then, therapy [22].

- Rescue of many thera(g)nosis processes described but formally out of this field, that is, there are many papers in which nanothera(g)nosis is not found in the title, abstracts and keywords, although they are really in this field.

- Nanotheragnostic robots based on the incorporation of auto-propulsion systems to the nanocarriers involved in Nanothera(g)nostic processes [46] will open a very exciting new scenario in this field. 
- Increasing quality assurance in both, the preparation of individual theragnostic agents and theragnostic nanoparticles as well as the diagnosis step in the nanothera(g)nosis processes.

- Social Responsibility, as in any high potential technology [47], is of great relevance applied to Nanothera(g)nosis because it represents the combination of key features (accountability, transparency, ethics and respect to the human rights and law) all of them oriented to fulfills the expectative and needs of the main stakeholders: the patients which should receive the principal benefits of the nanothera $(\mathrm{g})$ nostic treatment in preference of the interests of hospitals, governments, pharmaceutical multinationals, etc. which should be also taken into account but in second or third degree of importance.

\section{Conclusion}

As stated at the beginning of this paper, the main goal of it is to sending a message to chemists to be aware of the opportunities and challenges of Chemistry in Nanothera(g)nosis processes looking for innovation, social and economic impact in interdisciplinary framework by breaking the traditional frontiers and creating new paradigms when knowledge is not enough to solve Humanity problems, in this case the welfare of patients. This approach was proposed by Einstein at the beginning of $20^{\text {th }}$ Century.

A last remark is necessary for clarification purposes. The fact that "Nanotheragnostics" is an attractive keyword which nowadays opens many doors, it is incorrectly applied to describe processes which exclusively involves nanotherapies or nandiagnostics in isolation. The abuse of lightening words (e.g "sensors" in the 90s) is a frequent situation in scientific literature that must be systematically avoided.

\section{References}

1. Webster T (2012) Nanomedicine: technologies and applications, Elsevier, Amsterdam.

2. Smith LA, Ma PX (2004) Nanofibrous scaffolds for tissue engineering. Colloids and Interfaces B 39: 125-131

3. Bhunia SK, Maity SA, Ray SC, Jana NR (2013) Carbon nanoparticles-based fluorescent bioimaging probes. Scientific Reports 3:1473.

4. Santos HA, Mäkila HAE, Airaksinen AJ, Bimbo LM, Hirvonen J (2014) Porous silicon nanoparticles for nanomedicine: preparation and biomedical applications. Nanomedicine 9: 535-554.

5. Hedsden S (2017) World's first implantable artificial kidney could enter human trials.

6. Singh K, Panghal M, Kadyan S, Chaudhary U, Yadar JP (2014) Antibacterial Activity of Synthesized Silver Nanoparticles from Tinospora cordifolia against Multi Drug Resistant Strains of Pseudomonas aeruginosa Isolated from Burn Patients. Nanomed. Nanothechnol. 5: 4-6.

7. Lee $\mathrm{KH}$ (2014) On the use of nanocellulose as reinforcement of polymer matrix composites. Com Sci Tech 106: 15-27.

8. Whitesides JPA (2015) Reinventing chemistry. Angew Chemie Int Ed 54: 2-16.

9. Selvan ST, Narayanan K (2016) Introduction to Nanotheranostics Springer. Heildeberg.

10. Yaday SK (2016) Nanoscale Materials in Targeted Drug Delivery. Theragnosis and Tissue Regeneration Springer. Heidelberg.

11. Devasena T (2017) Therapeutic and Diagnostic Nanomaterials. Nanotheranosis journal, Springer.

12. Fan Z, Fu PP, Yu H, Ray PC (2014) Theragnostic nanomedicine for cancer detection and treatment. J Food Drug Anal 22: 3-17.

13. Lammers T, Aime II S, Hennink WE, Storm G, Kiessling F (2012) Theranostic Nanomedicne. Acc Chem Res 44: 1029-1038.
14. Wang K, Tang Z, Yang CJ, Kim Y, Fang X, et al. (2009) Molecular engineering of DNA: Molecular beacons Angew Chem Int Ed 48: 856-870.

15. Yao YY, Gedda G, Girma WM, Yen CL, Ling YC, et al. (2017) Magnetic fluorescent carbon dots for nanotheranosis. ACS Appl Mat Interfaces 9: 13887 13898.

16. Gopinath P, Uday Kumar S, Matai I, Bhushan B, Malwal D, et al. (2015) Cancer Nanotheranostics. Springer, Heidelberg.

17. Chen H, Zhang W, Zhu G, Xie J, Chen X (2017) Rethinking Cancer Nanotheranostics. Nat Rev Mat 2.

18. Kulkarni A, Rao P, Natarajan S, Goldman A, Sabbisetti VS, et al. (2016) Reporter nanoparticle that monitors its anticancer efficiency in real time. Proc Natl Acad Sci 113: E2104-E2013.

19. Pedrosa P, Vinhas R, Fernandes A, Baptista PV, et al. (2015) Gold nanotheragnostics: Proof-of-concept of Clinical Tool? Nanomaterials (Basel) 5: $1853-1879$.

20. Key J, Kim YS, Tatulli F, Palange AL, O'Neill B, et al. (2014) Opportunities for Nanoteragnosis in lung cancer and pulmonary metastasis. Clin Tras Imaging 2: $427-437$.

21. Veiseh O, Tang BC, Whitehead KA, Anderson DG, Langer R (2015) Managing diabetes with nanomedicine challanges and oportunities. Nat Rev Drug Dis 14: 45-57.

22. Bolognesi ML (2017) From Imaging Agents to Theragnostics drugs fo Alzheimer disease. Comprehensive Medicinal Chemistry III (Elsevier) 74-106.

23. Skaat H, Corem-Slakmon E, Grinberg I, Last D, Goez D, et al. (2013) Antibody-conjugated, dual mode, NIR fluorescent iron oxide nanoparticles for antiamyloidgenic activity and specific detection of amyloid- $\beta$-fibrils. Int $\mathrm{J}$ Nanomedicine 8: 4063-4076.

24. Busquets MA, Sabaté R, Estelrich J (2014) Potential applications of magnetic particles to detect and treat Alzheimer's disease. Nanoescale Research Letters 2: $538-548$.

25. Kouyoumdjian $\mathrm{H}$, Zhu DC, El-Dakdouki MH, Lorenz K, Chen J, et al. (2013) Glyconanoparticle Aided Detection of $\beta$ Amyloid by Magnetic Resonance Imaging and Attenuation of $\beta$ Amyloid Induced Cytotoxicity. ACS Chem Neurosci 4: 575-584.

26. Kim JW, Shashkov EV, Galanzha EI, Kotagirl W, Zharov LP (2007) Photothermal antimicrobial nanotherapy and Nanodiagnostics with self-assembling Carbon Nanotubes Clusters. Lasers Surg Med 39: 622-634.

27. Gelaperina S, Kisish K, Isenian MD, Heifets L (2005) The potential advantages of nanoparticle drug delivery systems in chemotherapy of tuberculosis. Am J Resp Crit Care Med 172: 1487-1490.

28. Mouhieddine TH, Itani MM, Nokkati A, Ren C, Daoud G, et al. (2015) Nanotheragnostic Applications for Ischemic and Hemorrhagic Strokes: Improved Delivery for a Better Prognosis. Curr Neurol Neurosci Rep 15: 505.

29. Reddy LH, Couvreur P (2011) Nanotechnology for therapy and imaging of liver diseases. J Hepatol 55: 1461-1466.

30. Matlin S, Mehta G, Hopf H (2015) Chemistry embraced by all. Science 347: 1179-1180.

31. Valcárcel M, López-Lorente Al, López-Jiménez MA (2017) Foundations of Analytical Chemistry. Springer, Heidelberg.

32. López-Lorente Al, Sieger M, Valcárcel M, Mizaikoff B (2014) Infrared attenuated total reflection spectroscopy for characterization of gold nanopartivles in solution. Anal Chem 86: 783-778.

33. Valcárcel M, López-Lorente Al (2014) Gold Nanoparticles in Analytical Chemistry. Elsevier Amsterdam.

34. Dong W, Le Y, Shi J (2011) Facile synthesis of monodispersed superparamagnetic $\mathrm{Fe}_{3} \mathrm{O}_{4}$-core@hybrid@Au-shell nanocomposite for bimodal imaging and photothermal therapy. Adv Mater 392-5397.

35. Killops KL, Campos LM, Hawker VJ (2008) Robust, Efficient, and Orthogonal Synthesis of Dendrimers via Thiol-ene "Click Chemistry. J Am Chem Soc 130: 5062-5064.

36. Conde L, Oliva N, Artzi N (2015) Implantable hydrogel embedded dark-gold nanoswitch as atheranostic probe to sense and overcome cancer multidrug resistance. Proc Natl Acad Sci 112: E1278-E1287. 
37. Choi $\mathrm{CH}$, Alabi CA, Webster $\mathrm{P}$, Davis ME (2010) Mechanism of active targeting in solid tumors with transferrin-containing gold nanoparticles. Proc Natl Acad Sci 107: 1235-1240.

38. De Leo V, Catucci L, Falqui A, Marotta R, Striccoli M, et al. (2014) Hybrid assemblies of fluorescent nanocrystals and membrane proteins in liposomes. Langmuir 30: 1599-1608.

39. Wenklaviak BW, Koch M, Hadjicostas E (2010) Quality assurance in analytical chemistry. Springer Heidelberg.

40. Kunjachan S, Ehling J, Storm G, Kiessling F, Lammers T (2015) Non-invasive Imaging of nanomedicines and nanotheranostics: Principles, progress and prospects. Chem Rew 115: 10907-10937.

41. Valcárcel M, Simonet BM (2008) Types of analytical information and their mutual relationships. Trends Anal Chem 27: 490-499.
42. Valcárcel M (2016) Quo vadis, analytical chemistry. Anal Bioanal Chem 408: 13-21.

43. Goodman SN, Fanelli D, loannidis JPA (2016) what does research reproducibility means? Science Translational Medicine 8: 341.

44. loainnidis JPA (2016) Why most clinical research is not useful? PLOS Med.

45. Ricotti L, Menciassi A (2015) Nanothecnology in biorobotics: opportunities and challenges. J Nanopart Res 17: 84-95.

46. Krogsgaard-Larsen $P$, Thostrup P, Besenbacher $F(2011)$ scientific socia responsibility: a call to arms. Angew Chem Int Ed 50: 2-4

47. Soriano ML, Zougagh M, Valcárcel M, Rios A (2018) Analytical nanoscience and nanotechnology: Where we are and where are heading. Talanta, 177: 104-121. 